\title{
A Modeling Framework for the Planning of Strategic Supply Chain Viewed from Complex Network
}

\author{
Jiangbo Zheng \\ School of management, Jinan University, Guangzhou, China. \\ E-mail: zhengjbjnu@126.com \\ Received February $18^{\text {th }}, 2009$; revised April 1 $1^{\text {st }}, 2009$; accepted May $4^{\text {th }}, 2009$.
}

\begin{abstract}
Based on the theory of complex network, this paper focuses on the planning of logistics nodes for strategic supply chain. I propose a practical mathematical modeling framework that simultaneously captures many practical aspects but still understated in the existing literatures of network planning problems. Moreover, capacity expansion and reduction scenarios are also analyzed as well as modular capacity shifts for the fluctuation of demands. So this paper is of importance for the research of network planning in strategic supply chain systems.
\end{abstract}

Keywords: strategic supply chain, logistics nodes, complex network

\section{Introduction}

With the increasingly competitive situation, enterprises have to face the changes of their internal operation mode and external supply chain mode for higher efficiency and lower operational costs. Different from traditional supply chains, a strategic supply chain emphasizes on the optimization of all resources and the coordinate development of the allies based on their core competences. What's more, a strategic supply chain is also different from the integrated supply chain, which mainly emphasizes on the overall control of the upstream and downstream of the supply chain and unilaterally pursues the stability of the chain. Therefore, strategic supply chain is a neutral competitive mode which focuses on the integration of allies' core competences and is of such great features as coordination, agility and difference. Under strategic supply chain mode, the coordination among the allies is more intimate and more dynamic. So the whole supply chain is of much more complexity and perhaps the traditional theories and methods can not give the further support for some relative research.

Complex network is a theory attempted to describe the properties of an actual or virtual network and to establish a model (for instance, mathematical model) to mirror those properties. Based on this theory and from the view of logistics nodes planning of a strategic supply chain, this paper proposes a mathematical modeling framework that captures many practical aspects of network design problems which have not been received adequate attention in the relative literatures. Such aspects considered include: dynamic planning horizon, generic supply chain network structure, external supply of materials, inventory opportunities for goods, distribution of commodities, facility configuration, availability of capital for investments, and storage limitations. Therefore, the research of this paper may provide some references for the mechanism of a strategic supply chain.

\section{The Significance for Strategic Supply Chain Design Applying Complex Network Theory}

To better understand the significance for strategic supply chain design, it is necessary to briefly review the background and the fundamental contents about complex network theory. A network is a set of items, which are called nodes, with connections between them, called edges. Systems taking the form of networks (also called graphs in some of mathematical literatures) abound in the world. Typical examples of networks include the World Wide Web, information networks of citations between academic papers, technological networks, biological networks and social networks of acquaintance or other connections between individuals, organizations and business relations among companies, and supply chain networks for sure. The study of networks, in the form of mathematical graph theory, is one of the fundamental pillars of discrete mathematics. Networks have also been studied extensively in the social sciences and in the 1930s socialists realized the importance of the patterns of 
connection between people to the understanding of the function of human society [1]. From then on, typical relevant researches address issues of centrality (which individuals are best connected to others or have most influence) and connectivity (whether and how individuals are connected to one another through the network). Recent years there have been a new change with the research focus shifting from the analysis of single small system and the property of individual nodes or edges within such systems to consideration of large-scaled (maybe millions or eve more of nodes and edges) statistics properties of systems. Recent work in this area is inspired particularly by a groundbreaking paper by Watts and Strogatz [2]. This new approach has been driven largely by the availability of computers and communication networks that allow us to gather and analyze data on a scale further larger than previously possible. This change of scale forces us a corresponding change in our analytic approach - strategic supply chain network is the case in point. For example, traditional research works about supply chain network of tens or hundreds nodes, it is relatively straightforward matter to draw a picture of the network with actual points and lines, and to give specific analysis (the human eye is also an analytic tool) about it through examining this picture. But this approach is not useful with a complex network of thousands or even more nodes - that is very common now for some modern multi-national companies' supply chain networks.

Furthermore, the theoretical body of complex network is established to do primary three aspects: 1) to find and highlight statistical properties, such as path lengths and degree distributions, which characterize the structure and behavior of a network, and to suggest appropriate ways to measure these properties; 2 ) to create models of networks that can help us to understand the meaning of these properties such as how they came to be as they are and how they interact with one another; 3) to predict what the behavior of networks system will be on the basis of measured structural properties and the local rules governing individual nodes. In fact, the scientific field has made an excellent start on the first two of these aims by drawing on ideas from a broad variety of disciplines. But such achievements are not well introduced in the research field of supply chain systems; especially the item (2) is understated in the planning of strategic supply chain network. Therefore, this paper attempts to apply complex networks theory to establish a modeling framework to better describe and interpret the systems of strategic supply chain.

\section{Literatures Review and Properties Description of a Strategic Supply Chain}

Research works on optimization of the supply chain through efficient planning decisions have been processed for many years such as Erlenkotter D. [3], Fong CO. and Srinivasan V. [4,5], Jacobsen SK [6], Sweeney DJ and Tatham RL [7] and in the strategic planning level, typical decisions concern the location of manufacturing and/or warehousing logistics nodes $[8,9,10]$. Moreover, recent years many research works have addressed the dynamic location problem such as: Daskin et al. [11] propose an extensive review of location problems, Beamon [12] distinguishes models with deterministic data from those with stochastic data, Owen and Daskin [13] clearly separate the static and dynamic models. Cordeau et al. [14] propose a static model considering a multi-commodity, multi-facility and single-country network. The decision variables concern the number of locations, the capacity and technology of manufacturing in plants and warehouses, selection of suppliers, selection of distribution channels, transportation modes and material flows. Hamer-Lavoie and Cordeau [15] simplify this model by removing the suppliers and the bills of materials. They suppose that the location has already been chosen for plants and the model focuses on warehouse location. The model is dynamic with stochastic demands, and takes inventories into account, including the safety stock. Dias et al. [16] work on the re-engineering of a two-echelon network (facilities and customers). The authors suppose that facilities can be opened, closed and reopened more than once during the planning horizon. They study these conditions within three scenarios: with maximum capacity restrictions; with both maximum and minimum capacity restrictions; and with a maximum capacity that decreases. With the same flexibility idea, Melo et al. [17] aim at relocating the network with expansion/reduction capacity scenarios. Despite of so many important researching works, some important real world issues have not received adequate attention. These include the external supply of products, inventory opportunities for products, storage limitations, availability of capital for investments, and relocation, expansion or reduction of nodes' capacities. Even though some of these issues have been researched individually in the literature, it is ignored that the structure of a network is strongly affected by the simultaneous consideration of these and other practical needs. One observes a lack of reasonably simple, yet comprehensive, models which can illustrate the effect of such factors on network configuration decisions.

In this paper, a supply chain is defined as a network of nodes (e.g. suppliers, manufacturing plants, distribution centers, warehouses, etc.) and lines (including physical lines, e.g. transportation lines, and virtual lines, e.g. information and communication channels) that perform a set of operations ranging from the acquisition of raw materials, the transformation of these materials into intermediate and finished products, to the distribution of the finished products to the customers network (Figure 1). 
supplier manufacturer distributor retailer customer

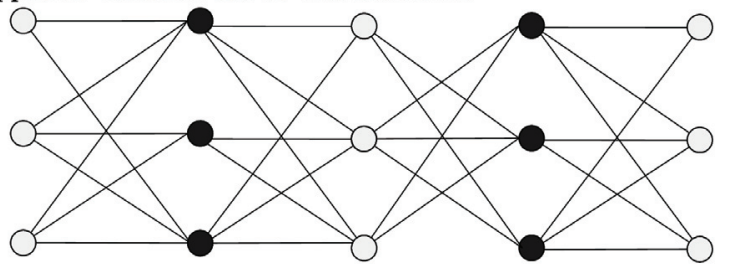

Figure 1. A network of supply chain

This paper attempts to bridge the gap between the complicated dynamic strategic supply chain in practice and the sound describing models in theory. So the main contribution is to provide a mathematical modeling framework for assisting decision-makers in the design of their supply chains. It is necessary to point out that the mathematical modeling framework in this paper firstly includes the relocation of existing logistics nodes, and can reflect the expansion and reduction of the nodes - it is very common in the practical supply chain operations. Further more, the notion of production in this paper is a wide conception which includes service and production. Then, the setup or shutdown of a node, or the expansion or reduction of a node, is usually a time-and-cost-consuming process. Finally, in strategic supply chain circumstances, it is emphasized that resources should be made full use and be integrated for better efficiency. So the capacity should be transmit to new nodes when some existing ones are shut down. Therefore, to implement smooth transition to a new network configuration needs better coordination of all operational aspects involved in this process, and better management of the required investment capital. Hence, to abate the financial burden put on such a comprehensive project, capital expenditures and network design decisions should be planned over some periods. Based on such contents, this paper tries to put forward a modeling framework which can generally reflect the factors taken into account in optimizing strategic supply chain.

\section{Notation and Definition of Decision Variables}

In order to simply describe the principle of the problem and precisely reflect the planning of logistics nodes in the supply chain, there is a very important disposal in this paper. That is, the planning horizon is partitioned into a set of consecutive and integer time periods which may not necessarily be equal. In total, there are $n$ planning periods. Then, it is assumed that if capacity is to be shifted then this will occur at the beginning of period $t$ ( $t \in\{1,2, \cdots, n\})$, and will have a relatively short duration compared to the period length. Finally, it is assumed that prior to the planning all relevant data (costs, capacities, and other factors) were collected through appropriate forecasting methods or company-specific business analyses.

\subsection{Sets}

It is defined that:

$N$ : set of logistics nodes.

$\varphi$ : set of selectable logistics nodes, $\varphi \subset N$.

$\varphi^{s}$ : set of selectable existing logistics nodes, $\varphi^{s} \subset \varphi$.

$\varphi^{n}$ : set of potential sites for establishing new logistics nodes, $\varphi^{n} \subset \varphi$.

$\mathfrak{R}$ : set of product types.

According to such definition, set $N$ contains all types of logistics nodes and these are categorized in so-called selectable and non-selectable logistics nodes which can be denoted as " $N \backslash \varphi$ ". Selectable logistics nodes form the set $\varphi$, a subset of $N$, and include existing logistics nodes $\varphi^{s}$ and potential sites for establishing new logistics nodes $\varphi^{n}$. Note that $\varphi^{s} \cap \varphi^{n}=\varphi, \varphi^{s}+\varphi^{n}=\varphi$. Another important assumption is: at the beginning of the planning horizon, all the logistics nodes in the set $\varphi^{s}$ are operating. Afterwards, capacity can be shifted from these logistics nodes to new nodes located at the sites.

\subsection{Costs}

It is defined that:

$P C_{m, p}^{t}$ : Variable cost of purchasing or producing one unit of product $p \in \mathfrak{R}$ by node $m \in N$ in period $t$.

$T C_{l, m, p}^{t}$ : variable cost of shipping one unit of product $p \in \mathfrak{R}$ from node 1 to node $\mathrm{m}(1, m \in N, l \neq m)$ in period $t$.

$I C_{m, p}^{t}$ : variable inventory carrying cost per unit on hand of product $\mathrm{p}$ in node $\mathrm{m}$ at the end of period $t$.

$M C_{i, j}^{t}$ : unit variable cost of moving capacity from the existing node $i \in \varphi^{s}$ to a new established node $j \in \varphi^{n}$ at the beginning of period $t$.

$O C_{m}^{t}$ : fixed cost of operating node $\mathrm{m}$ in period $t$.

$S C_{i}^{t}$ : fixed cost charged in period t for having shut down the existing node $i \in \varphi^{s}$ at the end of period $(t-1)$.

$F C^{t}{ }_{j}$ : fixed setup cost charged in period t when a new facility established at node $j \in \varphi^{n}$ starts its operation at the beginning of period $t+1$. 


\subsection{Important Parameters}

During the research of this paper, there are such important parameters:

$\bar{K}_{m}^{t}$ : maximum allowed capacity at node $\mathrm{m}$ in period $\mathrm{t}$ (similarly, $\underline{K}_{m}^{t}$ is the minimum required capacity at node $\mathrm{m}$ in period $\mathrm{t}$ ).

$\mu_{m, p}$ : unit capacity consumption factor of product $\mathrm{p}$ at node $\mathrm{m}$ in period $\mathrm{t}$.

$I_{m, p}$ :stock of product $\mathrm{p}$ of node $\mathrm{m}$ at the beginning of the planning horizon, observe that $I_{j, p}=0$ for $j \in \varphi^{n}$.

$B^{t}$ : available budget in period $\mathrm{t}$ where interest rate is $\lambda$, and assume that $\lambda$ is a constant.

It is necessary to emphasize on that since each existing node $i \in \varphi^{s}$ may have its capacity transferred to one or more new nodes, it is assumed that its maximum capacity is non-increasing during the planning horizon, that is, $\bar{K}_{i}^{t} \geqslant \bar{K}_{i}^{t+1}$.Similarly, potential new nodes must have non-decreasing capacities during the planning horizon, that is, $\bar{K}_{j}^{1}=0$ for $j \in \varphi^{n}$, and $\bar{K}_{j}^{t} \geqslant \bar{K}_{j}^{t+1}$.

\subsection{Decision Variables}

The decision variables of this paper are as follows:

$A_{m, p}^{t}$ : amount of product $p$ produced or purchased from an outside supplier by node $m \in N$ in period $t$. $x_{l, m, p}^{t}$ : amount of product $p$ shipped from node 1 to node $\mathrm{m}(1, m \in N, l \neq m)$ in period $t$.

$y_{m, p}^{t}$ : amount of product $p$ held in stock in node $m \in N$ at the end of period $t$.

$z_{i, j}^{t}$ : amount of capacity shifted from the existing node $i \in \varphi^{s}$ to a newly established node $j \in \varphi^{n}$ at the beginning of period $t$.

$\xi^{t}$ : capital not invested in period $\mathrm{t}$.

$\eta_{m}^{t}$ : It's a "0-1" variable, if the selectable node $m \in N$ is operated during period $t$, then $\eta_{m}^{t}=1$; otherwise, $\eta_{m}^{t}=0$.

According to such definition, it's obvious that $z_{i, j}^{1}=0$ because the capacity is not transferred at the beginning of planning horizon. What's more, at that time all existing nodes are operating and new nodes aren't established, so $\eta_{j}^{1}=0, \eta_{i}^{1}=1$.

\section{Mathematical Modeling Framework and the Interpretation}

\subsection{Mathematical Modeling Framework}

The formulation of this paper's model is as follows:

$$
\begin{aligned}
\min \omega= & \sum_{t} \sum_{m \in N} \sum_{p \in \Re} P C_{m, p}^{t} \cdot A_{m, p}^{t}+\sum_{t} \sum_{l \in N} \sum_{m \in N \backslash\{l\}} \sum_{p \in \Re} T C_{l, m, p}^{t} \cdot x_{l, m, p}^{t}+\sum_{t} \sum_{m \in N} \sum_{p \in \Re} I C_{m, p}^{t} \cdot y_{m, p}^{t} \\
& +\sum_{t} \sum_{m \in \varphi} O C_{m}^{t} \cdot \eta_{m}^{t}+\sum_{t} \sum_{m \in N \backslash \varphi} O C_{m}^{t}+\sum_{t} \sum_{i \in \varphi^{s}} S C_{i}^{t}+\sum_{t} \sum_{j \in \varphi^{n}} F C_{j}^{t}
\end{aligned}
$$

The formulation shows that the goal of planning horizon is to minimum the total costs. Where, the total costs are consist of purchasing/producing cost, shipping cost, inventory holding cost, operating cost, shutdown cost and establishing cost. The constraints are as follows (constraints of variables for non-negativity and integrality conditions are omitted):

$$
\begin{aligned}
& \text { S. t. } \\
& A_{m, p}^{t}+\sum_{m \in N\{\{l\}} x_{l, m, p}^{t}+y_{m, p}^{t-1}=D_{m, p}^{t}+\sum_{m \in N \backslash\{l\}} x_{m, l, p}^{t}+y_{m, p}^{t}, \quad l \in N \\
& \bar{K}_{i}^{1}-\sum_{\tau=1}^{t} \sum_{j \in \varphi^{n}} z_{i, j}^{\tau} \geq \bar{K}_{i}^{t} \cdot \eta_{i}^{t}, \quad i \in \varphi^{s} \\
& \sum_{\tau=1}^{t} \sum_{i \in \varphi^{s}} z_{i, j}^{\tau} \leq \bar{K}_{j}^{t} \cdot \eta_{j}^{t}, \quad j \in \varphi^{n} \\
& \sum_{\tau=1}^{t} \sum_{j \in \varphi^{n}} z_{i, j}^{\tau} \leq \bar{K}_{i}^{1}, \quad i \in \varphi^{s}
\end{aligned}
$$




$$
\begin{aligned}
& \sum_{p \in \Re} \mu_{i, p} \cdot\left(A_{i, p}^{t}+\sum_{l \in N \backslash\{i\}} x_{l, i, p}^{t}+y_{i, p}^{t-1}\right) \leq \bar{K}_{i}^{1}-\sum_{\tau=1}^{t} \sum_{j \in \varphi^{n}} z_{i, j}^{\tau}, \quad i \in \varphi^{s} \\
& \sum_{p \in \Re} \mu_{j, p} \cdot\left(A_{j, p}^{t}+\sum_{l \in N \backslash\{i\}} x_{l, j, p}^{t}+y_{j, p}^{t-1}\right) \leq \sum_{\tau=1}^{t} \sum_{i \in \varphi^{s}} z_{i, j}^{\tau}, \quad j \in \varphi^{n} \\
& \sum_{p \in \Re} \mu_{m, p} \cdot\left(A_{m, p}^{t}+\sum_{l \in N \backslash\{m\}} x_{l, m, p}^{t}+y_{m, p}^{t-1}\right) \leq \bar{K}_{m}^{t}, \quad m \in N \backslash \varphi \\
& \sum_{p \in \Re} \mu_{m, p} \cdot\left(A_{m, p}^{t}+\sum_{l \in N \backslash\{m\}} x_{l, m, p}^{t}+y_{m, p}^{t-1}\right) \geq \underline{K}_{m}^{t} \cdot \eta_{m}^{t}, \quad m \in \varphi \\
& \eta_{i}^{t} \geq \eta_{i}^{t+1}, \quad i \in \varphi^{s} \\
& \eta_{j}^{t} \geq \eta_{j}^{t+1}, j \in \varphi^{n} \\
& \sum_{j \in \varphi^{n}} F C_{j}^{1} \cdot \eta_{j}^{2}+\xi^{1}=B^{1} \\
& \sum_{i \in \varphi^{s}} \sum_{j \in \varphi^{n}} M C_{i, j}^{t} \cdot z_{i, j}^{t}+\sum_{i \in \varphi^{s}} S C_{i}^{t} \cdot\left(\eta_{i}^{t-1}-\eta_{i}^{t}\right)+\sum_{j \in \varphi^{n}} F C_{j}^{t} \cdot\left(\eta_{j}^{t+1}-\eta_{j}^{t}\right)+\xi^{t}=B^{t}+\lambda \cdot \xi^{t-1} \\
& \sum_{i \in \varphi^{s}} \sum_{j \in \varphi^{n}} M C_{i, j}^{n} \cdot z_{i, j}^{n}+\sum_{i \in \varphi^{s}} S C_{i}^{n} \cdot\left(\eta_{i}^{n-1}-\eta_{i}^{n}\right)+\xi^{n}=B^{n}+\lambda \cdot \xi^{n-1}
\end{aligned}
$$

\subsection{Interpretations of the Modeling Framework}

Constraint (2) is the usual demand-supply flow conservation conditions which must hold for each product, logistics node, and period.

Constraints $(3-5)$ ensure that only feasible capacity relocations can take place during the planning horizon. Where, constraint (3) guarantees that only operating existing logistics node $i \in \varphi^{s}$ can have their capacity moved to new facilities; constraint (4) imposes that by period t a new node has been constructed at site $j \in \varphi^{n}$ in order for a potential capacity relocation; constraint (5) states that if the capacity of an existing node has been completely transferred to others then the node has to be closed. The combination of (3) and (5) ensures that if an existing logistics node doesn't operate in a given period then its entire capacity was removed in one of the previous periods. Moreover, by constraint (5) no more capacity can be shifted out at the beginning of the planning horizon.

Constraints $(6-8)$ impose that the capacity of each node can't be exceeded in each period. Observe that constraint (6) also prevents any supply chain activities from taking place in existing nodes whose capacity has been totally relocated.

Constraint (9) state that it is only worth to operate a selectable node if its output is above a given minimum level. Constraints (10) and (11) allow the configuration of each selectable node to change at most once. Hence, if an existing node is closed, it can't be re-opened. Similarly, when a new node is established it will remain in operation until the end of the planning horizon.

Conditions $(12-14)$ are budget constraints. In each period, there is a limited amount of capital that can be spent on capacity transfers, shutting down existing nodes and establishing new logistics nodes. So the amount is given by the budget initially available in that period (represented by $\mathrm{B}^{\mathrm{t}}$ ) plus the remained capital not invested in previous periods (represented by $\lambda \cdot \xi^{t-1}$ ). In the first period, the allowed investments are as setting up new facilities that will start operating at the beginning of the second period (see constraint (12)). Moreover, in each one of the following period $t$, the available capital may cover capacity transfers, the costs incurred by closing existing logistics at the end of period $t-1$, and setups of new nodes that start operating at the beginning of period $t+1$ (see constraint (13)). In the last period $\mathrm{n}$, the allowed investments concern capacity transfers as well as shutdowns of nodes that ceased operating at the end of period $n-1$ (see constraint (14)).

Thus, through reasonable assumptions and simplified methods, this paper puts forward a practical mathematical modeling framework that is very easy to be solved by normal software. In fact, this model is a mixed-integer planning problem, and after given relational data or parameters we can obtain the results. So it's not necessary to give a computational experience. 


\section{Numerical Simulation by Computer}

A simple numerical example simulated by computer software is given in this part to prove the correctness and efficiency of my model. The objective function and relative restrains described in the previous sections were implemented using the modeling language ILOG OPL Studio 3.6, and a variety of test problems were solved with standard mathematical programming software, namely with the branch-and-bound algorithm of ILOG CPLEX 8.0, on a computer with the hardware of AMD Athlon(tm) 64X2 Dual PC with $1.8 \mathrm{GHz}$ processor and 1GB RAM.

In order to simulate the dynamic supply chain network circumstances and indefinite original quantities of logistics nodes, I randomly generated a tri-echelon network with various alternatives for the flow of products such as inter-facility transportation, direct deliveries from the manufacturer to the customer level, and reverse arcs for the return of goods (Figure 2). For such a problem, Table 1 indicates the number of facilities selected and non-selected and displays the average, minimum, and maximum CPU times (in seconds) required to attain optimality of this problem for various variants of the original model. Some important contents should be noted: the second column of Table 1 gives the number of problems considered in this kind of network (for details about the problem characteristics we refer the reader to relative literatures). Five instances were randomly generated for this problem, thus yielding a total of 20 instances. The generated problems cover small and medium-scale networks. In an attempt to generate problems related to realistic cases, the amount of arcs available for the transportation of goods in the networks is restricted to $60 \%$. Also, only a given number of product types can actually flow through each generated arc. In this way, we can constrain the volume of traffic in the networks. In all the problems, the relocation decisions involve the distribution centers and retailers. Furthermore, all data (costs, capacities, demands, etc.) were drawn at random from a uniform distribution over given intervals. These intervals were selected in such a way that a large variety of instances was created that differ by the number of periods, products and facilities, the availability of transportation arcs, the range of fixed and variable costs, and the range of capacities and capital for investments. For each subsequent period, the previous parameter value was increased or decreased by a certain percentage (for instance, $8 \%$ ) which was randomly selected over a given interval. For example, customer demands for the first period were drawn following a uniform distribution in the interval $[0,25]$. In the second period, the percentage increase compared to the first period was randomly generated by $8 \%$. This procedure was repeated until the last period of the planning horizon was attained, thus creat- ing an increasing demand sequence. As mentioned above, all the details can be found in Melo et al. [18].

\section{Conclusions}

Aiming at the planning problem of logistics nodes in strategic supply chain as well as through reasonable assumption and deep analysis, this paper makes research on it systematically. Based on the references of existing research woks and their insufficiencies, this paper establishes a mixed integer programming modeling framework for strategic supply chain design from the viewpoint of the planning of logistics nodes. The aspects considered include the relocation of existing nodes through

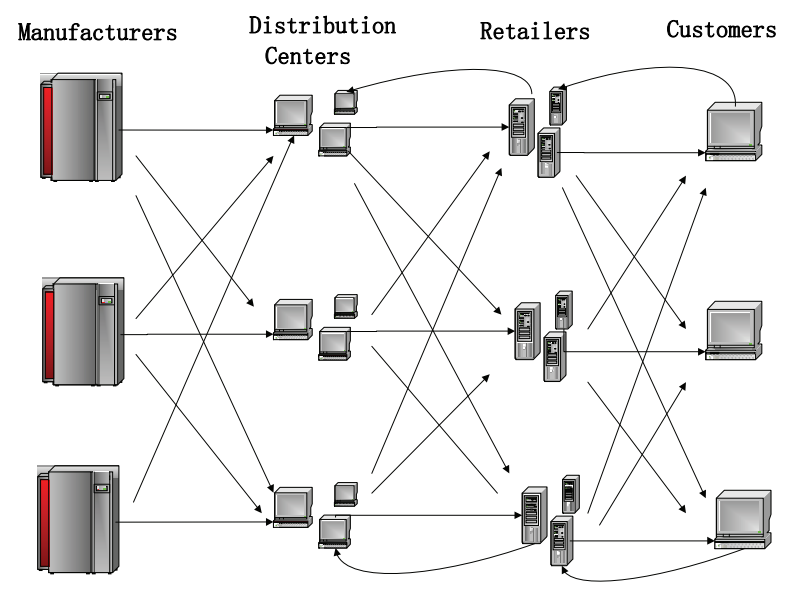

Figure 2. A tri-echelon network generated for computer simulation

Table 1. The results of computer simulation

\begin{tabular}{|c|c|c|c|}
\hline \multicolumn{3}{|l|}{ Problems } & 4 \\
\hline \multicolumn{3}{|l|}{ Periods } & $3,4,5$ \\
\hline \multicolumn{3}{|l|}{ Products } & 5 \\
\hline \multirow{2}{*}{$\begin{array}{l}\text { Non-selectable } \\
\text { facilities }\end{array}$} & \multicolumn{2}{|c|}{ Manufactures } & 5 \\
\hline & \multicolumn{2}{|c|}{ Customers } & 50,75 \\
\hline \multirow{4}{*}{$\begin{array}{l}\text { Selectable } \\
\text { facilities }\end{array}$} & \multirow{2}{*}{$\begin{array}{l}\text { Existing } \\
\text { facilities }\end{array}$} & DCs & 8 \\
\hline & & Retailers & 12 \\
\hline & \multirow{2}{*}{$\begin{array}{l}\text { New } \\
\text { facilities }\end{array}$} & DCs & 4 \\
\hline & & Retailers & 8 \\
\hline \multirow{3}{*}{ Variables } & \multicolumn{2}{|l|}{ Average } & 26268 \\
\hline & \multicolumn{2}{|l|}{ Minimal } & 14568 \\
\hline & \multicolumn{2}{|l|}{ Maximal } & 34204 \\
\hline \multirow{3}{*}{ Constraints } & \multicolumn{2}{|l|}{ Average } & 1968 \\
\hline & \multicolumn{2}{|l|}{ Minimal } & 1363 \\
\hline & \multicolumn{2}{|l|}{ Maximal } & 2315 \\
\hline \multirow{3}{*}{ CPU time(s) } & \multicolumn{2}{|l|}{ Average } & 234 \\
\hline & \multicolumn{2}{|l|}{ Minimal } & 21 \\
\hline & \multicolumn{2}{|l|}{ Maximal } & 1246 \\
\hline
\end{tabular}


capacity transfers to new locations, integration of inventory, transportation and supply decisions, the availability of a given budget for investments in node location and relocation, and the generic structure of the supply chain network. This paper has shown that capacity expansion and reduction scenarios as well as modular capacity shifts can easily be incorporated into this model. To verify the efficiency and correctness of the model, a computational simulation is generated by software. The simulation results show that a number of randomly generated test problems can be solved to optimality within no more than 2 hours at average. Therefore, my model is worthy of being applied for the dynamic situation and complex supply chain design.

In future research, an important extension for this model is expected to change the assumption of deterministic demand, costs, and other factors in the problem to stochastic variables. However, such a modification would have an impact on the complexity of the problem. So it is necessary to develop efficient solution methods to this very realistic and strategically significant practical problem.

\section{REFERENCES}

[1] J. L. Moreno, "Who shall survive," Beacon House, Beacon, NY, 1934.

[2] D. J. Watts and S. H. Strogatz, "Collective dynamics of 'small world' networks," Nature, No. 393, pp. 440-442, 1998.

[3] D. Erlenkotter, "A comparative study of approaches to dynamic location problems," European Journal of Operational Research, Vol. 6, pp. 133-143, 1981.

[4] C. O. Fong and V. Srinivasan, "The multi-region dynamic capacity expansion problem-Parts I and II," Operations Research, No. 29, pp. 787-816, 1981.

[5] C. O. Fong and V. Srinivasan, "The multi-region dynamic capacity expansion problem: An improved heuristic," Management Science, No. 32, pp. 1140-1152, 1986.

[6] S. K. Jacobsen, "Heuristic procedures for dynamic plant location," Technical Report of the Institute of Mathematical Statistics and Operations Research, 1977.
[7] D. J. Sweeney and R. L. Tatham, "An improved long-run model for multiple warehouse location," Management Science, No. 22, pp. 748-758, 1976.

[8] T. Bender, H. Hennes, J. Kalcsics, M. T. Melo, and S. Nickel, "Location software and interface with GIS and supply chain management," Berlin, Heidelberg: Springer, 2002.

[9] J. Kalcsics, T. Melo, S. Nickel, and V. Schmid-Lutz, "Facility location decisions in supply chain management," Operations Research Proceedings, Vol. 3, pp. 467-472, 2000.

[10] Q. Wang, R. Batta, J. Bhadury, and C. M. Rump, "Budget constrained location problem with opening and closing of logistics nodes," Computers and Operations Research, No. 30, pp. 2047-2069, 2003.

[11] M. Daskin, L. Snyder, and R. Berger, "Facility location in supply chain design," Logistics Systems: Design and Optimization, pp. 39-65, 2005.

[12] B. M. Beamon, "Supply chain design and analysis: Models and methods," International Journal of Production Economics, No. 55, pp.281-294, 1998.

[13] S. H. Owen and M. S. Daskin, "Strategic facility location: A review," European Journal of Operational Research, No. 111, pp. 423-447, 1998.

[14] J.-F. Cordeau, F. Pasin, and M. M. Solomon, "An integrated model for logistics network design," Annals of Operations Research, 144(1), pp. 59-82, 2006.

[15] G. Hamer-Lavoie and J. F. Cordeau, "Un mode'le pour la conception d'un re'seau de distribution avec localisation, affectation et stocks," INFOR, No. 44, pp. 99-115, 2006.

[16] J. Dias, M. E. Captivo, and J. Climaco, "Capacitated dynamic location problems with opening, closure and reopening of facilities," IMA Journal of Management Mathematics, Vol. 17, No. 4, pp. 317-348, 2006.

[17] D. Vila, A. Martel, and R. Beauregard, "Designing logistics networks in divergent process industries: A methodology and its application to the lumber industry," International Journal of Production Economics, 102(2), pp. 358-378, 2006.

[18] M. T. Melo, S. Nickel, and F. Saldanha da Gama, "Large-scale models for dynamic multi-commodity capacitated facility location," Technical Report 58, Fraunhofer Institute for Industrial Mathematics (ITWM) Kaiserslautern, Germany, 2003. 